\section{Bandagensystem erlaubt Patienten das Selbstmanagement}

— In Deutschland ist seit Kurzem ein innovatives Kompressionsbandagen-System zur Behandlung von venösen Ulzera erhältlich, das dank seiner einfachen Handhabbarkeit auch vom Patienten angelegt werden kann. Kompressionsmaßnahmen sind einer der tragenden Pfeiler in der Therapie von venösen Beinulzera. Wie Prof. Dr. Joachim Dissemond, Essen, erläuterte, greift die Kompression über die Einengung der dilatierten Venen und über die Steigerung des Gewebedrucks kausal in das Krankheitsgeschehen ein. „Mittels der Kompressionsbehandlung wird die Beschwerdesymptomatik verbessert, die Abheilung der Geschwüre unterstützt und ein Fortschreiten der Erkrankung verhindert", so der Dermatologe.

Defizite bei der Umsetzung: Das Anlegen eines Kompressionsverbands bedarf einer gewissen Übung und Erfahrung, damit der Verband weder zu fest noch zu locker sitzt und genau den erforderlichen Druck auf das Bein ausübt. Dass dies auch erfahrenen Pflegekräften oft nicht gelingt, hat eine Studie gezeigt, in der unter Kompressionsverbänden, die ambulant zur Behandlung des Ulcus cruris angelegt worden waren, Druckwerte zwischen 11 und 80 $\mathrm{mmHg}$ gemessen wurden. Die gewünsch- ten optimalen Druckwerte zwischen 30 und $50 \mathrm{mmHg}$ wurden im günstigsten Fall bei Verwendung von Zweikomponenten-Systemen von nur $27 \%$ der Pflegekräfte erreicht. Verschiedene Untersuchungen haben ergänzend belegt, dass lediglich ein geringer Teil der Patienten mit entsprechendem Bedarf auch tatsächlich eine Kompressionstherapie verordnet bekommt. „Aus diesen Beobachtungen lässt sich schließen, dass das Wissen um diese physikalische Behandlungsmethode sowohl bei Pflegekräften als auch bei Ärzten zunehmend verloren geht", schlussfolgerte Dissemond.
Einfache Kompressionsversorgung: Mit circaid $^{\varpi}$ juxtacures $^{\circledast}$ ist in Deutschland ein innovatives Kompressionsbandagen-System zur Behandlung von venösen Ulzera verfügbar, zu dessen wichtigsten Pluspunkten seine einfache Handhabung zählt. Das Anlegen der Versorgung ist so unkompliziert, dass diese Aufgabe nach Einweisung durch Fachpersonal leicht vom Patienten selbst übernommen werden kann. Das circaid $^{\circledR}$ Built-In-Pressure ${ }^{\circledR}$ system (BPS) ermöglicht das eigenständige Einstellen des verordneten Kompressionsniveaus sowie dessen Nachjustierung an sich verändernde Beinumfänge.

(wed)

Verlagsworkshop "Kompressionstherapie" (Veranstalter: Medical Data Institute, Starnberg)
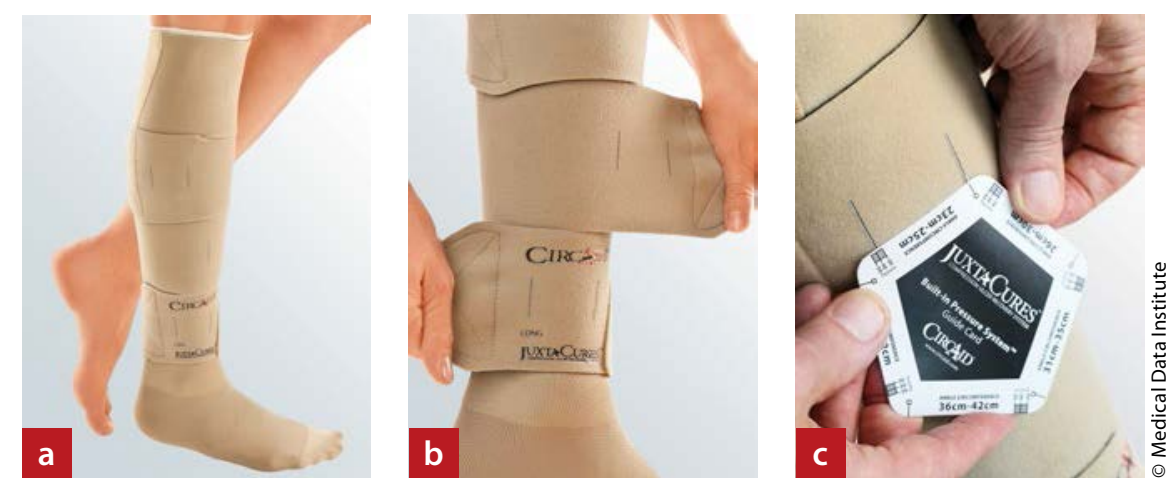

Das Kompressionsbandagensystem (a) ist einfach vom Patienten selbst anzulegen (b) und das Kompressionsniveau kann eigenständig eingestellt und nachjustiert werden (c).
Erweitertes Produktportfolio

\section{Therapieangebot bei Lymphödemen}

— Mit der Übernahme des Unternehmens FarrowMed einschließlich der patentierten FarrowWrap ${ }^{\circledast}$ ergänzt BSN medical sein Produktportfolio zur Versorgung von lymphatischen und Gefäßerkrankungen. Von dieser Übernahme profitieren sowohl BSN als auch unsere Kunden, so der Medizinprodukteanbieter. Damit könne man den immer zahlreicheren Patienten mit Lymphödemen und Wunden, ausgelöst durch chronische Gefäßinsuffizienz, noch umfassender und wirkungsvoller helfen.

www.bsnmedical.de

\section{Lymphödem \\ Pflege für strapazierte Haut}

— Bei Lymph- oder Lipödemen benötigt die Haut schonenden Pflege. Juzo Lymph Care wurde speziell für die tägliche Anwendung bei Lymph- und Lipödemen sowie Venenleiden entwickelt. Das Produkt ist frei von Konservierungsstoffen und enthält $4 \%$ Harnstoff. Damit spendet die Lotion wohltuende Feuchtigkeit und schützt die Haut vor dem Austrocknen. Sie ist speziell zur Pflege von strapazierter, trockener und schuppender Haut nach dem Tragen von Kompressionsbekleidung gedacht.

www.juzo.com
Armödem

\section{Lagerungshilfe unterstützt Entstauung}

— Die Behandlung von Lymphödemen im Arm- und Handbereich wird durch Hochlagerung der betroffenen Extremität begünstigt. Das Compressana Lymphkissen ist eine wertvolle Behandlungsunterstützung im Rahmen der Komplexen Entstauungstherapie (KPE). Daneben ist der Einsatz in der Schmerz- und Wundtherapie möglich. Bei konsequenter Anwendung der Lagerungshilfe kann neben der Unterstützung der Patientenbehandlung, der Therapie- und Rehabilitationszeitraum verkürzt werden.

www.compressana.de 